\title{
Uniform rotundity in every direction of Orlicz-Sobolev spaces
}

\author{
Fayun $\mathrm{CaO}^{1,2}$, Rui $\mathrm{Mao}^{1 *}$ and Bing Wang ${ }^{1}$
}

${ }^{*}$ Correspondence:

maorui1111@163.com

${ }^{1}$ Department of Mathematics, East China Normal University, Shanghai, 200241, China

Full list of author information is available at the end of the article

\begin{abstract}
In this paper, we study the extreme points and rotundity of Orlicz-Sobolev spaces. Analyzing and combining the properties of both Orlicz spaces and Sobolev spaces, we get the sufficient and necessary criteria for Orlicz-Sobolev spaces equipped with a modular norm to be uniformly rotund in every direction.
\end{abstract}

Keywords: Orlicz-Sobolev spaces; rotund; modular norm

\section{Introduction}

Sobolev spaces are valuable mathematical models which were formed in the 20th century. Their effect on the development of partial differential equations is obvious (see [1]). Orlicz-Sobolev spaces are an important extension of the Sobolev spaces, which have some properties of both Orlicz spaces and Sobolev spaces. Moreover, the development of OrliczSobolev spaces not only improves the Banach space theory but also provides a rich model for solving partial differential equations problems. The rotundity and uniformly rotundity in every direction are important geometric properties of Banach spaces. One of the reasons is that these properties are strongly related to the fixed point property (see [2]) and to the approximation theory (see [3, 4]). Zizler proved that if a normed linear space is uniformly rotund in every direction then it has a certain formal structure (see [5]).

Chen and Hu discussed the extreme points and rotundity of Orlicz-Sobolev spaces with maximum norm and Luxemburg norm (see [6, 7]). Garkavi first proposed the concept of uniform rotundity in every direction when he researched the center of Chebyshev (see [8]).

The modular norm of Orlicz-Sobolev spaces is the natural generalization of the Luxemburg norm. Then we can study the Orlicz-Sobolev spaces with modular norm by using the methods of Orlicz spaces. So we equip the Orlicz-Sobolev spaces with a modular norm and study Orlicz-Sobolev spaces.

In the second section we introduce some basic notions, we agree on terminology and provide some results which we will use further in the paper. In the third section we give some relationships between a modular and the modular norm. Furthermore we discuss the extreme points and give the sufficient and necessary criteria for Orlicz-Sobolev spaces equipped with a modular norm to be uniformly rotund in every direction.

\section{Preliminaries}

In this section we recall some basic notions and results. Let $[X,\|\cdot\|]$ be a Banach space, $S(X)$ the unit sphere and $B(X)$ the unit ball.

(c) Cao et al. 2016. This article is distributed under the terms of the Creative Commons Attribution 4.0 International License (http://creativecommons.org/licenses/by/4.0/), which permits unrestricted use, distribution, and reproduction in any medium, provided you give appropriate credit to the original author(s) and the source, provide a link to the Creative Commons license, and indicate if changes were made. 
Definition 2.1 ([9]) $M: \Re \rightarrow[0, \infty)$ is called an $N$ function if it has the following properties:

(1) $M$ is even, continuous, convex and $M(0)=0$.

(2) $M(u)>0$ for all $u \neq 0$.

(3) $\lim _{u \rightarrow 0} \frac{M(u)}{u}=0$ and $\lim _{u \rightarrow \infty} \frac{M(u)}{u}=\infty$.

Besides we call $M$ strictly convex if, for any $u \neq v$, we have

$$
M\left(\frac{u+v}{2}\right)<\frac{M(u)+M(v)}{2} .
$$

An interval $[a, b]$ is called a structural affine interval of $M$ provided that $M$ is affine on $[a, b]$, and it is not affine on either $[a-\varepsilon, b]$ or $[a, b+\varepsilon]$ for all $\varepsilon>0$. Let $\left\{\left[a_{i}, b_{i}\right]\right\}_{i}$ be all the structural affine intervals of $M$. We call

$$
S C_{M} \triangleq \Re \backslash\left[\bigcup_{i}\left(a_{i}, b_{i}\right)\right]
$$

the set of strictly convex points of $M$.

Proposition 2.1 ([9]) Suppose $M$ is strictly convex, then, for any $D>0, \varepsilon>0$, there exists $\delta>0$ such that, for any $u, v$, satisfying $|u| \leq D,|v| \leq D,|u-v| \geq \varepsilon$, we have

$$
M\left(\frac{u+v}{2}\right) \leq(1-\delta) \frac{M(u)+M(v)}{2} .
$$

Let $(\Omega, \Sigma, \mu)$ be the Lebesgue measure space in $\mathrm{p}$ Euclidean space with $0<\mu \Omega<\infty$ and $M$ be an $N$ function. For any measurable function $u$ on $\Omega$, we define the modular of $u$ by

$$
\rho(u)=\int_{\Omega} M(u(t)) d t .
$$

The Orlicz space is defined as follows:

$$
L_{M}:=L_{M}(\Omega)=\{u(t): \exists k>0, \rho(k u)<\infty\} .
$$

Then $L_{M}$ equipped with the Luxemburg norm

$$
\|u\|_{(M)}=\inf \left\{\lambda>0: \int_{\Omega} M\left(\frac{u(t)}{\lambda}\right) d t \leq 1\right\}
$$

becomes a Banach space which is called the Orlicz space.

Definition 2.2 ([9]) We say that the $N$ function $M$ satisfies the condition $\Delta_{2}$ (we write $\left.M \in \Delta_{2}\right)$ if there exist $K>2$ and $u_{0} \geq 0$ such that $M(2 u) \leq K M(u)\left(|u| \geq u_{0}\right)$.

Definition 2.3 ([10]) Let $X$ be a Banach space, $x \in S(X)$. If $y, z \in B(X), y+z=2 x$ implies $x=y=z$, then $x$ is called an extreme point of $B(X)$. The set of extreme points of $B(X)$ is denoted by $\operatorname{ext} B(X) . X$ is said to be $\operatorname{rotund}(\mathrm{R})$ if $S(X)=\operatorname{ext} B(X)$. 
Definition 2.4 ([11]) Let $X$ be a Banach space. $X$ is called uniformly rotund in every direction (URED) if for any $z \in X,\left\{x_{n}\right\} \subset X,\left\|x_{n}\right\| \rightarrow 1,\left\|x_{n}+z\right\| \rightarrow 1$ and $\left\|2 x_{n}+z\right\| \rightarrow 2$ imply $z=0$ as $n \rightarrow \infty$.

Remark 2.1 By Definition 2.3 and Definition 2.4, we get URED $\Rightarrow R$.

Next, we introduce the Orlicz-Sobolev space, which is an expansion of the Orlicz space.

Definition 2.5 ([1]) Let $M$ be an $N$ function and $\Omega$ be bounded and connected open subset of $\Re^{n}$. The Orlicz-Sobolev space is defined as follows:

$$
W^{m} L_{M}:=W^{m} L_{M}(\Omega)=\left\{u \in L_{M}: D^{\alpha} u \in L_{M}, 0 \leq|\alpha| \leq m\right\}
$$

where $\alpha=\left(\alpha_{1}, \alpha_{2}, \ldots, \alpha_{n}\right),|\alpha|=\sum_{i=1}^{n} \alpha_{i}, \alpha_{i}(i=1,2, \ldots, n)$ are nonnegative integers, $D^{\alpha} u$ is the $\alpha$ th distributional derivative of $u$.

Suppose $u \in W^{m} L_{M}$, we define its convex modular with respect to $M$ by

$$
\tilde{\rho}_{M}(u)=\sum_{0 \leq|\alpha| \leq m} \int_{\Omega} M\left(D^{\alpha} u(t)\right) d t
$$

and define the modular norm by

$$
\|u\|_{m,(M)}=\inf \left\{\lambda>0: \tilde{\rho}_{M}\left(\frac{u}{\lambda}\right) \leq 1\right\}
$$

Then $\left(W^{m} L_{M},\|\cdot\|_{m,(M)}\right)$ is a Banach space.

\section{Main results}

In this section, we first of all discuss the relationships between modular and the modular norm which are similar to that of Orlicz spaces. The results are showed through Lemma 3.1 to Lemma 3.3. Furthermore, these lemmas will help us to study the geometric properties in this paper. Second, we will discuss the extreme points. In this part we get a sufficient criterion for a point to be an extreme point or not, combining the generating function $M$ and properties of both Orlicz spaces and Sobolev spaces. Finally we get the sufficient and necessary criteria for Orlicz-Sobolev spaces equipped with a modular norm to be uniformly rotund in every direction.

Lemma 3.1 Suppose $u \in W^{m} L_{M}$, then:

(1) $\tilde{\rho}_{M}(u) \leq 1 \Rightarrow\|u\|_{m,(M)} \leq 1, \tilde{\rho}_{M}(u)=1 \Rightarrow\|u\|_{m,(M)}=1$.

(2) $u \neq 0, \tilde{\rho}_{M}\left(\frac{u}{\|u\|_{m,(M)}}\right) \leq 1$.

(3) $\|u\|_{m,(M)} \leq 1 \Rightarrow \tilde{\rho}_{M}(u) \leq\|u\|_{m,(M)}$.

(4) $\|u\|_{m,(M)}>1 \Rightarrow \tilde{\rho}_{M}(u) \geq\|u\|_{m,(M)}$.

Lemma 3.2 Suppose $M \in \Delta_{2}$ and $u \in W^{m} L_{M}$, then $\tilde{\rho}_{M}(u)=1 \Leftrightarrow\|u\|_{m,(M)}=1$.

Lemma 3.3 Suppose $M \in \Delta_{2}$ and $u \in W^{m} L_{M}$, then: 
(1) $\forall \varepsilon \in(0,1), \exists \delta \in(0,1)$, such that $\tilde{\rho}_{M}(u) \leq 1-\varepsilon \Rightarrow\|u\|_{m,(M)} \leq 1-\delta$.

(2) $\forall \varepsilon \in(0,1), \exists \delta \in(0,1)$, such that $\tilde{\rho}_{M}(u)>1+\varepsilon \Rightarrow\|u\|_{m,(M)} \geq 1+\delta$.

The proofs of Lemma 3.1 to Lemma 3.3 are clear.

Lemma 3.4 ([12]) Let $\alpha=\inf \left\{t_{1}:\left(t_{1}, t_{2}, \ldots, t_{n}\right) \in \Omega\right\}, \beta=\sup \left\{t_{1}:\left(t_{1}, t_{2}, \ldots, t_{n}\right) \in \Omega\right\}, r \in$ $[\alpha, \beta]$. Assume that $f \in L_{M}$ satisfies $f(t) \geq 0$, for $\mu$-a.e. $t=\left(t_{1}, t_{2}, \ldots, t_{n}\right) \in \Omega$. Then iff $(t)$ is non-decreasing with respect to $t_{1}$ and $f(t)=0$ for $t_{1} \leq r$, we have $\left\|\int_{E(t)} f(t) d s\right\|_{(M)} \leq(\beta-$ $r)\|f\|_{(M)}$, where $E(t)=E_{r}\left(t_{1}, t_{2}, \ldots, t_{n}\right)=\left\{s \in\left[r, t_{1}\right]:\left(s, t_{2}, \ldots, t_{n}\right) \in \Omega\right\}$.

Base on the above lemmas, we obtain the following result.

Theorem 3.1 Suppose $u \in W^{m} L_{M}$, the following statements are equivalent:

(1) $M \in \Delta_{2}$.

(2) $\tilde{\rho}_{M}(u) \rightarrow 0 \Leftrightarrow\|u\|_{m,(M)} \rightarrow 0$.

(3) $\tilde{\rho}_{M}(u) \rightarrow 1 \Leftrightarrow\|u\|_{m,(M)} \rightarrow 1$.

Proof $(1) \Rightarrow(2)$. The proof is similar to that of the Orlicz spaces (see [1]).

$(2) \Rightarrow(1)$. Otherwise, if $M \notin \Delta_{2}$, then there exists a nonnegative sequence $\left\{c_{k}\right\} \uparrow \infty$ such that

$$
M\left(\left(1+\frac{1}{k}\right) c_{k}\right)>2^{k} M\left(c_{k}\right)
$$

Set $\lambda_{k}=\frac{1}{2^{k} M\left(c_{k}\right)}(k=1,2, \ldots), \alpha, \beta$ are defined as in Lemma 3.4. Without loss of generality, let $\sum_{k=1}^{\infty} \lambda_{k}<\mu(\Omega)$. Taking $\delta_{0} \in(\alpha, \beta)$ such that setting $\Omega_{0}=\left\{t \in \Omega: t_{1} \leq \delta_{0}\right\}$, we have $\mu\left(\Omega_{0}\right)=\mu(\Omega)-\sum_{k=1}^{\infty} \lambda_{k}$. Then choose $\delta_{1} \in\left(\delta_{0}, \beta\right)$ and $\Omega_{1}=\left\{t \in \Omega: \delta_{0}<t_{1} \leq \delta_{1}\right\}$ such that $\mu\left(\Omega_{1}\right)=\lambda_{1}$. By the induction process, for any $k \in N^{+}$, we have $\Omega_{k}=\left\{t \in \Omega: \delta_{k-1}<t_{1} \leq \delta_{k}\right\}$ such that $\mu\left(\Omega_{k}\right)=\lambda_{k}$. Obviously, $\sum_{k=0}^{\infty} \mu\left(\Omega_{k}\right)=\mu(\Omega)$ and $M\left(c_{k}\right) \mu\left(\Omega_{k}\right)=\frac{1}{2^{k}}$ for any $k \in N^{+}$.

Let

$$
u_{p}(t)=\sum_{k=p+1}^{\infty} c_{k} \cdot \chi_{\Omega_{k}}(t), \quad 1 \leq p<\infty
$$

then

$$
\begin{aligned}
\rho_{M}\left(u_{p}\right) & =\int_{\Omega} M\left(\sum_{k=p+1}^{\infty} c_{k} \cdot \chi_{\Omega_{k}}(t)\right) d t \\
& =\sum_{k=p+1}^{\infty} \int_{\Omega_{k}} M\left(c_{k} \cdot \chi_{\Omega_{k}}(t)\right) d t \\
& =\sum_{k=p+1}^{\infty} M\left(c_{k}\right) \cdot \mu\left(\Omega_{k}\right) \\
& =\sum_{k=p+1}^{\infty} \frac{1}{2^{k}}=\frac{1}{2^{p}},
\end{aligned}
$$

which implies $\left\|u_{p}\right\|_{(M)} \leq 1$. 
On the other hand, for any $l>1$, there exists $p_{0}$ satisfying $l \geq 1+\frac{1}{p_{0}}$, thus for all $p \geq p_{0}$,

$$
\begin{aligned}
\rho_{M}\left(l u_{p}\right) & \geq \rho_{M}\left(\left(1+\frac{1}{p_{0}}\right) u_{p}\right)=\sum_{k=p+1}^{\infty} M\left(\left(1+\frac{1}{p_{0}}\right) c_{k}\right) \cdot \mu\left(\Omega_{k}\right) \\
& \geq \sum_{k=p+1}^{\infty} M\left(\left(1+\frac{1}{k}\right) c_{k}\right) \cdot \mu\left(\Omega_{k}\right) \\
& \geq \sum_{k=p+1}^{\infty} 2^{k} M\left(c_{k}\right) \cdot \mu\left(\Omega_{k}\right)>1
\end{aligned}
$$

We have $\left\|u_{p}\right\|_{(M)} \geq \frac{1}{\frac{1}{l}}=l$, thus $\left\|u_{p}\right\|_{(M)} \geq 1$. Consequently, $\left\|u_{p}\right\|_{(M)}=1$.

Define

$$
\tilde{u}_{j, p}(t)=\int_{\alpha}^{t_{1}} \int_{\alpha}^{x_{j-1}} \cdots \int_{\alpha}^{x_{1}} \sum_{k=p+1}^{\infty} c_{k} \cdot \chi_{\Omega_{k}}\left(s, t_{2}, \ldots, t_{n}\right) d s d x_{1} d x_{2} \cdots d x_{j-1}, \quad 1 \leq j \leq m,
$$

$\tilde{u}_{0, p}(t)=u_{p}(t)$.

Then, for $\alpha=(j, 0,0, \ldots, 0)$, we have $D^{(j, 0,0, \ldots, 0)} \tilde{u}_{m, p}(t)=\tilde{u}_{m-j, p}(t), 0 \leq j \leq m$. And at the same time for $\alpha \notin\{(0,0, \ldots, 0),(1,0, \ldots, 0), \ldots,(m, 0, \ldots, 0)\}$,

$$
D^{\alpha} \tilde{u}_{m, p}(t)=0
$$

Furthermore, by the induction and Lemma 3.4, we derive

$$
\left\|D^{\alpha} \tilde{u}_{m, p}\right\|_{(M)} \leq\left(\beta-\delta_{p}\right)^{m-|\alpha|}\left\|u_{p}\right\|_{(M)}
$$

It follows from $\lambda_{k} \rightarrow 0$ that $\mu\left(\Omega_{k}\right)=\lambda_{k} \rightarrow 0, k \rightarrow \infty$. This implies that $\delta_{k}$ approaches $\beta$. We can conclude, for any $\alpha \neq(m, 0,0, \ldots, 0),\left\|D^{\alpha} \tilde{u}_{m, p}\right\|_{(M)} \rightarrow 0$ as $p \rightarrow \infty$. By (3.1) and Lemma 3.1(3), we obtain $\tilde{\rho}_{M}\left(\tilde{u}_{m, p}\right) \rightarrow 0, p \rightarrow \infty$. Thus $\left\|\tilde{u}_{m, p}\right\|_{m,(M)} \leq 1, p \rightarrow \infty$.

But

$$
\left\|\tilde{u}_{m, p}\right\|_{m,(M)} \geq\left\|u_{p}\right\|_{(M)}=1,
$$

since

$$
\left\{\lambda>0: \tilde{\rho}_{M}\left(\frac{\tilde{u}_{m, p}}{\lambda}\right) \leq 1\right\} \subset\left\{\lambda>0: \rho_{M}\left(\frac{u_{p}}{\lambda}\right) \leq 1\right\} .
$$

By the above discussion we have

$$
\tilde{\rho}_{M}\left(\tilde{u}_{m, p}\right) \rightarrow 0, \quad\left\|\tilde{u}_{m, p}\right\|_{m,(M)}=1, \quad p \rightarrow \infty
$$

It is a contradiction. Thus $M \in \Delta_{2}$.

$(1) \Rightarrow(3)$. By Lemma 3.1(3), (4) and Lemma 3.3.

$(3) \Rightarrow(1)$. Suppose $\tilde{\rho}_{M}(u) \rightarrow 1 \Leftrightarrow\|u\|_{m,(M)} \rightarrow 1$ and $M \notin \Delta_{2}$, by the proof of $(2) \Rightarrow(1)$ we can get

$$
\tilde{\rho}_{M}\left(\tilde{u}_{m, p}\right) \rightarrow 0, \quad\left\|\tilde{u}_{m, p}\right\|_{m,(M)}=1, \quad p \rightarrow \infty
$$

It is a contradiction. Thus $M \in \Delta_{2}$. 
Now, we turn to the extreme points and rotundity of Orlicz-Sobolev spaces.

Proposition 3.1 Suppose $u \in S\left(W^{m} L_{M}\right)$, if there exist $\varepsilon>0$ and affine intervals $\left(a_{\alpha}, b_{\alpha}\right)$ of $M, 0 \leq|\alpha| \leq m$ such that

$$
\text { int } \bigcap_{0 \leq|\alpha| \leq m}\left\{t \in \Omega: D^{\alpha} u(t) \in\left(a_{\alpha}+\varepsilon, b_{\alpha}-\varepsilon\right)\right\} \neq \emptyset,
$$

then $u \notin \operatorname{ext} B\left(W^{m} L_{M}\right)$.

Proof Let $\Omega_{0}=\bigcap_{0 \leq|\alpha| \leq m}\left\{t \in \Omega: D^{\alpha} u(t) \in\left(a_{\alpha}+\varepsilon, b_{\alpha}-\varepsilon\right)\right\}$. Take $t^{\prime}, t^{\prime \prime}, r>0$, such that $U\left(t^{\prime}, r\right) \subset \Omega_{0}, U\left(t^{\prime \prime}, r\right) \subset \Omega_{0}$, and $U\left(t^{\prime}, r\right) \cap U\left(t^{\prime \prime}, r\right)=\emptyset$, define

$$
\begin{aligned}
& J_{t^{\prime}}(t)= \begin{cases}e^{-\frac{1}{r^{2}-\sum_{i=1}^{n}\left(t_{i}-t_{i}^{\prime}\right)^{2}},} & t \in U\left(t^{\prime}, r\right), \\
0, & t \in \Omega \backslash U\left(t^{\prime}, r\right),\end{cases} \\
& J_{t^{\prime \prime}}(t)= \begin{cases}e^{-\frac{1}{r^{2}-\sum_{i=1}^{n}\left(t_{i}-t_{i}^{\prime \prime}\right)^{2}},} & t \in U\left(t^{\prime \prime}, r\right), \\
0, & t \in \Omega \backslash U\left(t^{\prime \prime}, r\right),\end{cases}
\end{aligned}
$$

then $J_{t^{\prime}}(t), J_{t^{\prime \prime}}(t) \in C_{c}^{\infty}(\Omega) \subset W^{m} L_{M}(\Omega)$.

Let

$$
\begin{aligned}
& c_{1}=\varepsilon \cdot \min _{0 \leq|\alpha| \leq m}\left\{\frac{1}{\max _{t \in \Omega}\left|D^{\alpha} J_{t^{\prime}}(t)\right|+1}\right\}, \\
& c_{2}=\varepsilon \cdot \min _{0 \leq|\alpha| \leq m}\left\{\frac{1}{\max _{t \in \Omega}\left|D^{\alpha} J_{t^{\prime \prime}}(t)\right|+1}\right\},
\end{aligned}
$$

then $c_{1}>0, c_{2}>0$, and $c_{1} D^{\alpha} J_{t^{\prime}}(t) \leq \varepsilon, c_{2} D^{\alpha} J_{t^{\prime \prime}}(t) \leq \varepsilon$, for any $\alpha$ with $0 \leq|\alpha| \leq m, t \in \Omega$. Let $c=\min \left\{c_{1}, c_{2}\right\}$. Then we define

$$
v(t)=u(t)+c J_{t^{\prime}}(t)-c J_{t^{\prime \prime}}(t), \quad w(t)=u(t)-c J_{t^{\prime}}(t)+c J_{t^{\prime \prime}}(t), \quad t \in \Omega,
$$

then $v, w \in W^{m} L_{M}, w+v=2 u$, and $w \neq v$. Let $M(u)=k_{\alpha} u+d_{\alpha}$ on $\left(a_{\alpha}, b_{\alpha}\right)$, therefore

$$
\begin{aligned}
\int_{\Omega} M\left(D^{\alpha} v(t)\right) d t= & \int_{\Omega \backslash U\left(t^{\prime}, r\right) \backslash U\left(t^{\prime \prime}, r\right)} M\left(D^{\alpha} u(t)\right) d t+\int_{U\left(t^{\prime}, r\right)} M\left(D^{\alpha} u(t)+D^{\alpha} c J_{t^{\prime}}(t)\right) d t \\
& +\int_{U\left(t^{\prime \prime}, r\right)} M\left(D^{\alpha} u(t)-D^{\alpha} c J_{t^{\prime \prime}}(t)\right) d t \\
= & \int_{\Omega \backslash U\left(t^{\prime}, r\right) \backslash U\left(t^{\prime \prime}, r\right)} M\left(D^{\alpha} u(t)\right) d t+\int_{U\left(t^{\prime}, r\right)}\left(k_{\alpha} D^{\alpha} u(t)+d_{\alpha}\right) d t \\
& +\int_{U\left(t^{\prime \prime}, r\right)}\left(k_{\alpha} D^{\alpha} u(t)+d_{\alpha}\right) d t \\
& +\int_{U\left(t^{\prime}, r\right)} k_{\alpha} D^{\alpha} c J_{t^{\prime}}(t) d t-\int_{U\left(t^{\prime \prime}, r\right)} k_{\alpha} D^{\alpha} c J_{t^{\prime \prime}}(t) d t \\
= & \int_{\Omega \backslash U\left(t^{\prime}, r\right) \backslash U\left(t^{\prime \prime}, r\right)} M\left(D^{\alpha} u(t)\right) d t+\int_{U\left(t^{\prime}, r\right) \cup U\left(t^{\prime \prime}, r\right)}\left(k_{\alpha} D^{\alpha} u(t)+d_{\alpha}\right) d t \\
= & \int_{\Omega} M\left(D^{\alpha} u(t)\right) d t .
\end{aligned}
$$


So we conclude $\tilde{\rho}_{M}(v)=\tilde{\rho}_{M}(u) \leq 1$. Likewise, $\tilde{\rho}_{M}(w)=\tilde{\rho}_{M}(u) \leq 1$. Therefore $v, w \in$ $B\left(W^{m} L_{M}\right)$ and $v \neq w$, we know that $u \notin \operatorname{ext} B\left(W^{m} L_{M}\right)$.

Lemma 3.5 Let $u \in S\left(W^{m} L_{M}\right)$, if

(i) $\tilde{\rho}_{M}(u)=1$, and

(ii) $\mu\left\{t \in \Omega: u(t) \notin S C_{M}\right\}=0$,

then $u \in \operatorname{ext} B\left(W^{m} L_{M}\right)$.

Proof Suppose $v, w \in B\left(W^{m} L_{M}\right)$ and $w+v=2 u$. We have to show $u=v=w$. The convexity of $M$ implies

$$
\begin{aligned}
1 & =\tilde{\rho}_{M}(u)=\sum_{0 \leq|\alpha| \leq m} \int_{\Omega} M\left(D^{\alpha} u(t)\right) d t \\
& =\sum_{0 \leq|\alpha| \leq m} \int_{\Omega} M\left(D^{\alpha}\left(\frac{v(t)+w(t)}{2}\right)\right) d t \\
& \leq \sum_{0 \leq|\alpha| \leq m}\left(\frac{1}{2} \int_{\Omega} M\left(D^{\alpha} v(t)\right) d t+\frac{1}{2} \int_{\Omega} M\left(D^{\alpha} w(t)\right) d t\right) \\
& =\frac{1}{2} \sum_{0 \leq|\alpha| \leq m} \int_{\Omega} M\left(D^{\alpha} v(t)\right) d t+\frac{1}{2} \sum_{0 \leq|\alpha| \leq m} \int_{\Omega} M\left(D^{\alpha} w(t)\right) d t \\
& \leq 1 .
\end{aligned}
$$

So the equality holds in the above inequations. Again by the convexity of $M$, for any $\alpha$ with $0 \leq|\alpha| \leq m$,

$$
\int_{\Omega} M\left(D^{\alpha} u(t)\right) d t=\frac{1}{2} \int_{\Omega} M\left(D^{\alpha} v(t)\right) d t+\frac{1}{2} \int_{\Omega} M\left(D^{\alpha} w(t)\right) d t
$$

In particular, when $\alpha=(0,0, \ldots, 0)$,

$$
\int_{\Omega} M(u(t)) d t=\frac{1}{2} \int_{\Omega} M(v(t)) d t+\frac{1}{2} \int_{\Omega} M(w(t)) d t .
$$

Then by (ii), we conclude $u=v=w$. So $u \in \operatorname{ext} B\left(W^{m} L_{M}\right)$ holds.

Theorem 3.2 Let $M$ be a strictly convex $N$ function. $W^{m} L_{M}$ is rotund if and only if $M \in \Delta_{2}$.

Proof Sufficiency. It is trivial by Lemma 3.2 and Lemma 3.5.

Necessity. Suppose $M \notin \Delta_{2}$, by the proof process of Theorem 3.1, there exist $p_{0}$ and $p_{0+1}$ such that

$$
\left\|\tilde{u}_{m, p_{0}}\right\|_{m,(M)}=1, \quad\left\|\tilde{u}_{m, p_{0+1}}\right\|_{m,(M)}=1 .
$$

Let $w=\tilde{u}_{m, p_{0}}, v=\tilde{u}_{m, p_{0+1}}$, and $u=\frac{w+v}{2}$.

Then

$$
\|u\|_{m,(M)}=1 .
$$


Therefore $v, w \in B\left(W^{m} L_{M}\right)$, but $w \neq v$, so $u \notin \operatorname{ext} B\left(W^{m} L_{M}\right)$. That is a contradiction with the fact that $W^{m} L_{M}$ is rotund. Thus the condition $M \in \Delta_{2}$ holds.

Based on the above results, we can prove the following theorem.

Theorem 3.3 Let $M$ be a strictly convex $N$ function. $W^{m} L_{M}$ is URED if and only if $M \in \Delta_{2}$.

Proof Sufficiency. Suppose $w \in W^{m} L_{M},\left\{u_{n}\right\} \subset W^{m} L_{M}$ satisfying $\left\|u_{n}\right\|_{m,(M)} \rightarrow 1$, $\| u_{n}+$ $w \|_{m,(M)} \rightarrow 1$ and $\left\|2 u_{n}+w\right\|_{m,(M)} \rightarrow 2$ as $n \rightarrow \infty$. Let $v_{n}=u_{n}+w$. We get

$$
\left\|v_{n}\right\|_{m,(M)} \rightarrow 1, \quad\left\|\frac{u_{n}+v_{n}}{2}\right\|_{m,(M)} \rightarrow 1 \quad \text { as } n \rightarrow \infty
$$

Furthermore $\tilde{\rho}_{M}\left(u_{n}\right) \rightarrow 1, \tilde{\rho}_{M}\left(v_{n}\right) \rightarrow 1, \tilde{\rho}_{M}\left(\frac{u_{n}+v_{n}}{2}\right) \rightarrow 1$, since $M \in \Delta_{2}$. Now we claim that $\left\{u_{n}-v_{n}\right\}$ converges in measure to 0 . Otherwise, there exist $\delta_{0}>0, \varepsilon_{0}>0$, and $\left\{u_{n_{k}}\right\}$ and $\left\{v_{n_{k}}\right\}$, such that

$$
\mu\left\{t \in \Omega:\left|u_{n_{k}}(t)-v_{n_{k}}(t)\right| \geq \delta_{0}\right\} \geq \varepsilon_{0}
$$

Since $\tilde{\rho}_{M}\left(u_{n_{k}}\right) \rightarrow 1$, for any $\overline{\varepsilon_{0}}$, choose $k_{0} \in N^{+}$, for $k \geq k_{0}$ we have

$$
\left|\tilde{\rho}_{M}\left(u_{n_{k}}\right)-1\right|<\overline{\varepsilon_{0}} \text {. }
$$

Then for $D=M^{-1}\left(\frac{3\left(1+\overline{\varepsilon_{0}}\right)}{\varepsilon_{0}}\right)$ and $k \geq k_{0}$,

$$
\begin{aligned}
1+\overline{\varepsilon_{0}} & >\tilde{\rho}_{M}\left(u_{n_{k}}\right)=\sum_{0 \leq|\alpha| \leq m} \int_{\Omega} M\left(D^{\alpha} u_{n_{k}}(t)\right) d t \\
& \geq \int_{\left\{t \in \Omega:\left|u_{n_{k}}(t)\right|>D\right\}} M\left(u_{n_{k}}(t)\right) d t \\
& \geq M(D) \mu\left\{t \in \Omega:\left|u_{n_{k}}(t)\right|>D\right\},
\end{aligned}
$$

which implies

$$
\mu\left\{t \in \Omega:\left|u_{n_{k}}(t)\right|>D\right\}<\frac{\varepsilon_{0}}{3} \quad\left(k \geq k_{0}\right) .
$$

Likewise, $\mu\left\{t \in \Omega:\left|v_{n_{k}}(t)\right|>D\right\}<\frac{\varepsilon_{0}}{3}\left(k \geq k_{0}\right)$.

Define

$$
\Omega_{D, \delta_{0}}=\left\{t \in \Omega:\left|u_{n_{k}}(t)-v_{n_{k}}(t)\right| \geq \delta_{0},\left|u_{n_{k}}(t)\right| \leq D,\left|v_{n_{k}}(t)\right| \leq D\right\} \quad\left(k \geq k_{0}\right) .
$$

Since

$$
\begin{aligned}
& \Omega_{D, \delta_{0}} \cup\left\{t \in \Omega:\left|u_{n_{k}}(t)\right|>D\right\} \cup\left\{t \in \Omega:\left|v_{n_{k}}(t)\right|>D\right\} \\
& \quad \supset\left\{t \in \Omega:\left|u_{n_{k}}(t)-v_{n_{k}}(t)\right| \geq \delta_{0}\right\} .
\end{aligned}
$$


Then the following inequalities hold:

$$
\begin{aligned}
\mu\left(\Omega_{D, \delta_{0}}\right) \geq & \mu\left(\left\{t \in \Omega:\left|u_{n_{k}}(t)-v_{n_{k}}(t)\right| \geq \delta_{0}\right\}\right)-\mu\left(\left\{t \in \Omega:\left|u_{n_{k}}(t)\right|>D\right\}\right) \\
& -\mu\left(\left\{t \in \Omega:\left|v_{n_{k}}(t)\right|>D\right\}\right) \\
\geq & \frac{\varepsilon_{0}}{3} .
\end{aligned}
$$

By Proposition 2.1, for the given $D, \delta_{0}$, there exists $\delta>0$ such that

$$
M\left(\frac{v_{n_{k}}(t)+u_{n_{k}}(t)}{2}\right)<(1-\delta) \frac{M\left(v_{n_{k}}(t)\right)+M\left(u_{n_{k}}(t)\right)}{2},
$$

where $t \in \Omega_{D, \delta_{0}}$.

Therefore

$$
\begin{aligned}
& 1=\lim _{k \rightarrow \infty} \tilde{\rho}_{M}\left(\frac{u_{n_{k}}+v_{n_{k}}}{2}\right)=\lim _{k \rightarrow \infty} \sum_{0 \leq|\alpha| \leq m} \int_{\Omega} M\left(D^{\alpha}\left(\frac{u_{n_{k}}(t)+v_{n_{k}}(t)}{2}\right)\right) d t . \\
& =\lim _{k \rightarrow \infty} \int_{\Omega} M\left(\frac{u_{n_{k}}(t)+v_{n_{k}}(t)}{2}\right) d t+\lim _{k \rightarrow \infty} \sum_{1 \leq|\alpha| \leq m} \int_{\Omega} M\left(D^{\alpha}\left(\frac{u_{n_{k}}(t)+v_{n_{k}}(t)}{2}\right)\right) d t \\
& =\lim _{k \rightarrow \infty} \int_{\Omega_{D, \delta_{0}}} M\left(\frac{u_{n_{k}}(t)+v_{n_{k}}(t)}{2}\right) d t+\lim _{k \rightarrow \infty} \int_{\Omega \backslash \Omega_{D, \delta_{0}}} M\left(\frac{u_{n_{k}}(t)+v_{n_{k}}(t)}{2}\right) d t \\
& +\lim _{k \rightarrow \infty} \sum_{1 \leq|\alpha| \leq m} \int_{\Omega} M\left(D^{\alpha}\left(\frac{u_{n_{k}}(t)+v_{n_{k}}(t)}{2}\right)\right) d t \\
& \leq \lim _{k \rightarrow \infty}(1-\delta) \int_{\Omega_{D, \delta_{0}}} \frac{M\left(v_{n_{k}}(t)\right)+M\left(u_{n_{k}}(t)\right)}{2} d t+\lim _{k \rightarrow \infty} \int_{\Omega \backslash \Omega_{D, \delta_{0}}} M\left(\frac{u_{n_{k}}(t)+v_{n_{k}}(t)}{2}\right) d t \\
& +\lim _{k \rightarrow \infty} \sum_{1 \leq|\alpha| \leq m} \int_{\Omega} M\left(D^{\alpha}\left(\frac{u_{n_{k}(t)}+v_{n_{k}}(t)}{2}\right)\right) d t \\
& \leq \lim _{k \rightarrow \infty} \frac{\rho_{M}\left(u_{n_{k}}\right)+\rho_{M}\left(v_{n_{k}}\right)}{2}-\lim _{k \rightarrow \infty} \delta \int_{\Omega_{D, \delta_{0}}} \frac{M\left(v_{n_{k}}(t)\right)+M\left(u_{n_{k}}(t)\right)}{2} d t \\
& +\lim _{k \rightarrow \infty} \sum_{1 \leq|\alpha| \leq m} \int_{\Omega} M\left(D^{\alpha}\left(\frac{u_{n_{k}}(t)+v_{n_{k}}(t)}{2}\right)\right) d t \\
& \leq \lim _{k \rightarrow \infty} \frac{\rho_{M}\left(u_{n_{k}}\right)+\rho_{M}\left(v_{n_{k}}\right)}{2}-\lim _{k \rightarrow \infty} \delta \int_{\Omega_{D, \delta_{0}}} M\left(\frac{u_{n_{k}}(t)-v_{n_{k}}(t)}{2}\right) d t \\
& +\lim _{k \rightarrow \infty} \sum_{1 \leq|\alpha| \leq m} \int_{\Omega} M\left(D^{\alpha}\left(\frac{u_{n_{k}}(t)+v_{n_{k}}(t)}{2}\right)\right) d t \\
& \leq \lim _{k \rightarrow \infty} \frac{\rho_{M}\left(u_{n_{k}}\right)+\rho_{M}\left(v_{n_{k}}\right)}{2}-\delta M\left(\frac{\delta_{0}}{2}\right) \mu\left(\Omega_{D, \delta_{0}}\right) \\
& +\lim _{k \rightarrow \infty} \sum_{1 \leq|\alpha| \leq m} \int_{\Omega} M\left(D^{\alpha}\left(\frac{u_{n_{k}}(t)+v_{n_{k}}(t)}{2}\right)\right) d t \\
& \leq \lim _{k \rightarrow \infty} \frac{\rho_{M}\left(u_{n_{k}}\right)+\rho_{M}\left(v_{n_{k}}\right)}{2}-\delta M\left(\frac{\delta_{0}}{2}\right) \mu\left(\Omega_{D, \delta_{0}}\right) \\
& +\lim _{k \rightarrow \infty} \sum_{1 \leq|\alpha| \leq m}\left[\frac{1}{2} \int_{\Omega} M\left(D^{\alpha} u_{n_{k}}(t)\right) d t+\frac{1}{2} \int_{\Omega} M\left(D^{\alpha} v_{n_{k}}(t)\right) d t\right]
\end{aligned}
$$




$$
\begin{aligned}
& \leq \lim _{k \rightarrow \infty} \frac{1}{2} \tilde{\rho}_{M}\left(u_{n_{k}}\right)+\lim _{k \rightarrow \infty} \frac{1}{2} \tilde{\rho}_{M}\left(v_{n_{k}}\right)-\delta M\left(\frac{\delta_{0}}{2}\right) \frac{\varepsilon_{0}}{3} \\
& \leq 1-\delta M\left(\frac{\delta_{0}}{2}\right) \frac{\varepsilon_{0}}{3}<1 .
\end{aligned}
$$

It is a contradiction. Then the claim holds, so $w=0$. We finished the proof of sufficiency. Necessity follows from Remark 2.1 and Theorem 3.2.

\section{Competing interests}

The authors declare that they have no competing interests.

\section{Authors' contributions}

All authors conceived of the study, participated in its design and coordination, drafted the manuscript, participated in the sequence alignment, and read and approved the final manuscript.

\section{Author details}

'Department of Mathematics, East China Normal University, Shanghai, 200241, China. ${ }^{2}$ Shanghai Key Laboratory of PMMP, East China Normal University, Shanghai, 200241, China.

\section{Acknowledgements}

This work is supported by the Science and Technology Commission of Shanghai Municipality (STCSM) under Grant No. $13 \mathrm{dz} 2260400$. The authors would like to thank the handling editors for the help in the processing of the paper. The authors are very grateful to the anonymous referees for the useful suggestions and remarks, which helped to improve the contents of this article.

Received: 1 June 2016 Accepted: 25 October 2016 Published online: 08 November 2016

\section{References}

1. Adams, RA: Sobolev Spaces. Academic Press, New York (1975)

2. Goebel, K, Kirk, WA: Topics in Metric Fixed Point Theory. Cambridge University Press, Cambridge (1990)

3. Odyniec, W, Lewicki, G: Minimal Projection in Banach Spaces. Lecture Notes in Mathematics. Springer, Berlin (1990)

4. Singer, I: The Theory of Best Approximation and Functional Analysis. SIAM, Philadelphia (1974)

5. Zizler, V: On some rotundity and smoothness properties of Banach spaces. Diss. Math. 87, 5-33 (1971)

6. Chen, ST, Hu, CY: On the extreme points and rotundity of Luxemburg norm in Orlicz Sobolev spaces. Nat. Sci. J. Harbin Norm. Univ. 17(2), 1-6 (2001) (in Chinese)

7. Chen, ST, Hu, CY: The extreme points and rotundity of Orlicz Sobolev spaces with maximum norm. J. Nat. Sci. Heilongjiang Univ. 18(4), 15-22 (2001) (in Chinese)

8. Garkavi, AL: On the Chebyshev center of a set in a normed space. J. Nat. Sci. Heilongjiang Univ., $328-331$ (1961) (in Russian)

9. Chen, ST: Geometry of Orlicz spaces. Diss. Math. 356, 1-204 (1996)

10. Ding, GG: Introduction to Banach Spaces. Science Press, Beijing (1984) (in Chinese)

11. Smith, MA: Some examples concerning rotundity in Banach spaces. Math. Ann., 233, 155-161 (1978)

12. Chen, ST, Hu, CY, Zhao, XJ: Uniform rotundity of Orlicz Sobolev spaces. Soochow J. Math. 29(3), $299-312$ (2003)

\section{Submit your manuscript to a SpringerOpen ${ }^{\circ}$ journal and benefit from:}

- Convenient online submission

Rigorous peer review

- Immediate publication on acceptance

- Open access: articles freely available online

- High visibility within the field

- Retaining the copyright to your article 